\title{
The exact WKB for cosmological particle production
}

\author{
Seishi Enomoto ${ }^{a}$ and Tomohiro Matsuda ${ }^{b}$ \\ ${ }^{a}$ School of Physics, San Yat-sen University, \\ No.135, Xingang Xi Road, Guangzhou 510275, China \\ ${ }^{b}$ Laboratory of Physics, Saitama Institute of Technology, \\ Fukaya, Saitama 369-0293, Japan \\ E-mail: seishi@mail.sysu.edu.cn, matsuda@sit.ac.jp
}

\begin{abstract}
The Bogoliubov transformation in cosmological particle production can be explained by the Stokes phenomena of the corresponding ordinary differential equation. The calculation becomes very simple as far as the solution is described by a special function. Otherwise, the calculation requires more tactics, where the Exact WKB (EWKB) may be a powerful tool. Using the EWKB, we discuss cosmological particle production focusing on the effect of more general interaction and classical scattering. The classical scattering appears when the corresponding scattering problem of the Schrödinger equation develops classical turning points on the trajectory. The higher process of fermionic preheating is also discussed using the Landau-Zener model.
\end{abstract}

KEYwords: Cosmology of Theories beyond the SM, Nonperturbative Effects

ArXiv ePrint: 2010.14835 


\section{Contents}

1 Introduction

1.1 Cosmological preheating as the scattering problem of the Schrödinger equation with inverted quadratic potential for $E>0$

1.2 Preheating as scattering with the quadratic potential $E<0 \quad 5$

2 The exact WKB for preheating $\quad 8$

2.1 Connection formulae by EWKB $\quad 9$

2.2 Exact WKB for $E>0$ inverted quadratic potential 13

$3 \quad$ EWKB for higher dimensional interaction $\quad 16$

$\begin{array}{lll}3.1 & \text { Quantum scattering for inverted quartic potential } & 16\end{array}$

$\begin{array}{lll}3.2 & \text { Classical scattering for inverted double-well potential } & 19\end{array}$

4 Fermions $\quad \mathbf{2 0}$

4.1 Landau-Zener model and kinematic particle creation in cosmology 20

4.2 Cosmological particle production of fermions 23

5 Conclusions and discussions $\quad 24$

$\begin{array}{ll}\text { A Comparison with the steepest descent method } & 25\end{array}$

\section{Introduction}

Particles may be formed from the vacuum when fundamental parameters such as mass or interaction coefficients become time-dependent, and there are many reasons for which the fundamental parameters could change during cosmological evolution. Among them, particle production caused by inflaton oscillation is known to be very important for solving the problem of reheating the universe after inflation [1-3].

The motion of inflaton field is a damped oscillation. However, at least near the center of the oscillation, where particle production is likely to take place, linear approximation with respect to $t$ can be made. Then, one can write the inflaton motion as $\phi(t)=v t$, which drastically simplifies the problem. Typically, the mass of a scalar field (e.g, $\chi$ ) is supposed to be given by

$$
m_{\chi}^{2}(t)=m_{0}^{2}+g_{2}^{2} \phi(t)^{2}
$$

where $\phi(t)$ is the oscillating inflaton field. If we consider the Lagrangian given by

$$
\mathcal{L}_{\chi}=\frac{1}{2} \partial_{\mu} \chi \partial^{\mu} \chi-\frac{1}{2} m_{0}^{2} \chi^{2}-\frac{1}{2} g_{2}^{2} \phi(t)^{2} \chi^{2}
$$


the equation of motion is given by

$$
\frac{d^{2} \chi}{d t^{2}}+\left[k^{2}+m_{\chi}^{2}(t)\right] \chi=0
$$

If one replaces $\phi(t)$ with $\phi(t) \simeq v t$, the above equation is equivalent to the Schrödinger equation of the scattering problem by the "inverted quadratic potential" given by

$$
V(t)=-\left(g_{2}^{2} v^{2}\right) t^{2}
$$

where the corresponding "energy" is

$$
E=k^{2}+m_{0}^{2} .
$$

Note that $E>V$ is always true in this case. Therefore, there is no classical turning point in the scattering problem. The particle formation (preheating) with $E<V$ is discussed in ref. [4] and succeeding papers. Sometimes the phenomenon is called tachyonic preheating, since $E<V$ is realized when $m_{\chi}^{2}<0$.

There are a variety of methods for finding the wave functions of the general onedimensional scattering problem of quantum mechanics. For the inverted quadratic potential, one can find the exact solution (i.e, the Weber function, or the parabolic cylinder functions). Alternatively, one can calculate the scattering coefficients approximating the potential by a series of steps [5]. This gives the integral of the coefficients. Analytic continuation of the WKB expansion and related topics have a long history [6]. For instance, it has been applied to pair production in vacuum by an alternating field [7]. The Schwinger mechanism [8], which is named after Schwinger who first derived the exponential formula for the pair production, can be analyzed using the complex $\mathrm{WKB}$, and is still an active research target $[9,10]$. For cosmological preheating, a similar calculation has been given by Chung in ref. [11]. In refs. [12, 13], the method has been used to calculate the effect of higher-dimensional interaction during preheating. In this paper, we consider the Exact WKB analysis (EWKB) $[14,15]$ for the particle formation with some exotic interactions. The EWKB is a powerful tool for calculating the connection formula, especially useful when the entire solution cannot be transformed into a special function. Using the EWKB, we analyze the effects of higher-dimensional interaction and the classical scattering of bosons and fermions. The reader can compare these results with our earlier calculation $[12,13]$ reviewed in appendix A. In our next paper, we are going to examine asymmetric particle formation (kinematic baryogenesis) using the results obtained in this paper [16].

\subsection{Cosmological preheating as the scattering problem of the Schrödinger equation with inverted quadratic potential for $E>0$}

Before using the EWKB method to cosmological preheating, let us remember how particle production has been treated in the typical cosmological preheating scenario. Here, we ignore the expansion factor of the universe for simplicity, since our model considers almost instant particle production and also the factor can be included by redefining the parameters. Typically, the WKB expansion is used to find

$$
\chi_{k}(t)=\frac{\alpha_{k}(t)}{\sqrt{2 \omega_{k}}} e^{-i \int^{t} \omega d t}+\frac{\beta_{k}(t)}{\sqrt{2 \omega}} e^{+i \int^{t} \omega d t},
$$


where

$$
\omega_{k}(t) \equiv k^{2}+m_{\chi}^{2}(t)
$$

We take $\alpha_{k}=1, \beta_{k}=0$ for the initial vacuum state. The distribution of the particle in the final state is

$$
n_{\chi}(k)=\left|\beta_{k}\right|^{2}
$$

which can be found by solving the scattering problem of the corresponding Schrödinger equation. For the above model (i.e, scattering by the inverted quadratic potential), the following Weber equation

$$
y^{\prime \prime}(z)+\left(\nu+\frac{1}{2}-\frac{1}{4} z^{2}\right) y(z)=0
$$

has the solution $D_{\nu}(z), D_{-\nu-1}(i z) .{ }^{1}$ More specifically, one can define

$$
z \equiv i e^{i \pi / 4} \sqrt{2 g_{2} v} t
$$

in the original field equation to find

$$
\frac{d^{2} \chi}{d z^{2}}+\left[\nu+\frac{1}{2}-\frac{1}{4} z^{2}\right] \chi=0
$$

Here we defined

$$
\nu=\frac{k^{2}+m_{0}^{2}}{2 g_{2} v} i-\frac{1}{2}
$$

and for later use we define

$$
\kappa \equiv \frac{k^{2}+m_{0}^{2}}{2 g_{2} v}
$$

and

$$
\nu=i \kappa-\frac{1}{2}
$$

Here, $\kappa$ is an important parameter, which is later used to estimate the particle production. The asymptotic forms are given by

$$
\begin{aligned}
\text { 1. } & |\arg z|<\frac{3 \pi}{4} & D_{\nu}(z) \rightarrow e^{-z^{2} / 4} z^{\nu} \\
\text { 2. } & -\frac{5}{4} \pi<\arg z<-\frac{\pi}{4} & D_{\nu}(z) \rightarrow e^{-\frac{z^{2}}{4}} z^{\nu}-\frac{\sqrt{2 \pi}}{\Gamma(-\nu)} e^{-i \nu \pi+\frac{z^{2}}{4}} z^{-\nu-1}, \\
\text { 3. } & \frac{\pi}{4}<\arg z<\frac{5 \pi}{4} & D_{\nu}(z) \rightarrow e^{-\frac{z^{2}}{4}} z^{\nu}-\frac{\sqrt{2 \pi}}{\Gamma(-\nu)} e^{i \nu \pi+\frac{z^{2}}{4}} z^{-\nu-1} .
\end{aligned}
$$

\footnotetext{
${ }^{1}$ Note that the following relation

$$
D_{\nu}(z)=e^{i \nu \pi} D_{\nu}(-z)+\frac{\sqrt{2 \pi}}{\Gamma(-\nu)} e^{i(\nu+1) \pi / 2} D_{-\nu-1}(-i z)
$$

tells that both $D_{\nu}(-z)$ and $D_{-\nu-1}(i z)$ are also the solutions of the equation, although they are not linearly independent.
} 
Since $z \equiv i e^{i \pi / 4} \sqrt{2 g_{2} v} t$ is used here, $t<0$ gives $\frac{5 \pi}{4}<\arg z<\frac{9 \pi}{4}$, which corresponds to the region 1 . Also, $t \rightarrow+\infty$ corresponds to the region 3 . Therefore, we find for $t \rightarrow-\infty$,

$$
\begin{aligned}
e^{-\frac{z^{2}}{4}} & =e^{-i \frac{g_{2} v}{2} t^{2}} \\
z^{\nu} & =e^{\left(i \kappa-\frac{1}{2}\right) \log z}=e^{\left(i \kappa-\frac{1}{2}\right)\left(\log \left(\sqrt{2 g_{2} v}|t|\right)+i \frac{3 \pi}{4}\right)},
\end{aligned}
$$

which gives $\left(t=-|t|=e^{\pi i}|t|\right.$ is used here)

$$
\begin{aligned}
D_{\nu}(z) & \simeq e^{-i \frac{g_{2} v}{2} t^{2}} e^{\left(i \kappa-\frac{1}{2}\right)\left(\log \left(\sqrt{2 g_{2} v}|t|\right)-i \frac{\pi}{4}\right)}, \\
D_{-\nu-1}(i z) & \simeq e^{+i \frac{g_{2} v}{2} t^{2}} e^{\left(-i \kappa-\frac{1}{2}\right)\left(\log \left(\sqrt{2 g_{2} v}|t|\right)+i \frac{\pi}{4}\right)} .
\end{aligned}
$$

Note that the above solutions in the limit $t \rightarrow-\infty$ are giving the \pm WKB solutions of eq. (1.6). Therefore, we define

$$
\begin{aligned}
& \chi_{-} \rightarrow D_{\nu}(z) \\
& \chi_{+} \rightarrow D_{-\nu-1}(i z) .
\end{aligned}
$$

On the other hand, in the $t \rightarrow+\infty$ limit we find

$$
\begin{aligned}
e^{-\frac{z^{2}}{4}} & =e^{-i \frac{g_{2} v}{2} t^{2}} \\
z^{\nu} & =e^{\left(i \kappa-\frac{1}{2}\right) \log z}=e^{\left(i \kappa-\frac{1}{2}\right)\left(\log \left(\sqrt{2 g_{2} v} t\right)+i \frac{3 \pi}{4}\right)},
\end{aligned}
$$

which gives in this limit,

$$
\begin{aligned}
D_{\nu}(z) \simeq & e^{-i \frac{g_{2} v}{2} t^{2}} e^{\left(i \kappa+\frac{1}{2}\right)\left(\log \left(\sqrt{2 g_{2} v} t\right)+i \frac{3 \pi}{4}\right)} \\
& +i \frac{\sqrt{2 \pi}}{\Gamma(-\nu)} e^{i \frac{g_{2} v}{2} t^{2}} e^{-\kappa \pi} e^{\left(-i \kappa-\frac{1}{2}\right)\left(\log \left(\sqrt{2 g_{2} v} t\right)+i \frac{3 \pi}{4}\right)} .
\end{aligned}
$$

Immediately, one will find that in the $t=+\infty$ limit the asymptotic form of the exact solution $D_{\nu}(z)$ is the mixture of the \pm WKB solutions, which gives the connection formula. In this case, the connection formula gives the Bogoliubov transformation of the WKB solutions. In the calculation of the connection formula, we use

$$
\begin{aligned}
\Gamma(z) \Gamma(1-z) & =\frac{\pi}{\sin \pi z} \\
\Gamma(\bar{z}) & =\overline{\Gamma(z)} \\
1+\nu & =1+\left(i \kappa-\frac{1}{2}\right)=-\bar{\nu}
\end{aligned}
$$

for the calculation of $\Gamma(-\nu)=\Gamma\left(-i \kappa+\frac{1}{2}\right)$. This gives

$$
\begin{aligned}
\left.\Gamma(-\nu)\right|^{2} & =\frac{\pi}{\sin \pi(-\nu)} \\
& =\frac{2 \pi i}{e^{-i \pi \nu}-e^{i \pi \nu}} \\
& =\frac{2 \pi}{e^{\pi \kappa}+e^{-\pi \kappa}} \\
\Gamma(-\nu) & =\frac{\sqrt{2 \pi} e^{-\pi \kappa / 2}}{\sqrt{1+e^{-2 \pi \kappa}}} e^{\arg \Gamma(-\nu)} .
\end{aligned}
$$


Finally, one obtains the connection formula given by

$$
\left(\begin{array}{c}
\alpha_{k}^{R} \\
\beta_{k}^{R}
\end{array}\right)=\left(\begin{array}{cc}
\sqrt{1+e^{-2 \pi \kappa}} e^{i \theta_{1}} & i e^{-\pi \kappa+i \theta_{2}} \\
-i e^{-\pi \kappa-i \theta_{2}} & \sqrt{1+e^{-2 \pi \kappa}} e^{-i \theta_{1}}
\end{array}\right)\left(\begin{array}{c}
\alpha_{k}^{L} \\
\beta_{k}^{L}
\end{array}\right),
$$

where $\mathrm{L}$ and $\mathrm{R}$ are for $t \rightarrow-\infty$ and $t \rightarrow+\infty$, respectively. Here, all the phase parameters are included in $\theta_{1,2}(k)$. Viewing the result as the solution of the scattering problem, the reflection and the penetration amplitudes are

$$
\begin{aligned}
\left|R_{k}\right| & =\frac{e^{-\pi \kappa}}{\sqrt{1+e^{-2 \pi \kappa}}} \\
\left|T_{k}\right| & =\frac{1}{\sqrt{1+e^{-2 \pi \kappa}}} .
\end{aligned}
$$

The above calculation can be obtained by approximating the potential by a series of steps. This alternative approach gives

$$
\begin{aligned}
& \dot{\alpha}_{k}=\beta_{k} \frac{\dot{\omega}_{k}}{2 \omega_{k}} e^{+2 i \int_{-\infty}^{t} d t^{\prime} \omega_{k}} \\
& \dot{\beta}_{k}=\alpha_{k} \frac{\dot{\omega}_{k}}{2 \omega_{k}} e^{-2 i \int_{-\infty}^{t} d t^{\prime} \omega_{k}},
\end{aligned}
$$

which can be integrated to give the connection formula [5, 11-13]. Note that the analytic continuation is possible for the above integration.

In the above (simple) scenario, there is no classical reflection point (turning point) on the real axis, which means that classically the reflection is not allowed in the scattering problem. Particle production becomes significant when $\kappa<1$, where the quantum scattering process becomes significant.

On the other hand, it would be natural to think about the effect of classical particle production caused by a classical turning point. Is the significant amplification of the particle production possible in this case? This is the primary question in this paper. The difference from the tachyonic preheating scenario will be discussed clearly in the text.

\subsection{Preheating as scattering with the quadratic potential $E<0$}

For later use, we analyze particle creation when $m_{\chi}^{2}(t)<0$ is realized temporary. The typical potential and the Stokes lines of the model are given in figure 1.

The situation is similar to the tachyonic preheating scenario after hybrid inflation [4]. However, if one identifies $\chi$ as the waterfall field of the hybrid inflation model, the field is already tachyonic from the beginning of the oscillation. ${ }^{2}$ In this case, one is not calculating the scattering by the classical turning point, since the starting point of the process is already between the two turning points(see figure 1) and the later process occurs within the tachyonic region. Note that the end of hybrid inflation is defined by $m_{\chi}\left(\phi_{c}\right)^{2}=0$, which corresponds to the turning point of figure 1 . (We are neglecting $k$ just for simplicity.) This is not the situation we are considering in this paper. We are considering the situation when

\footnotetext{
${ }^{2}$ There could be some exceptional models of hybrid inflation [17-19], which changes the conditions discussed in this section. Here, we will focus on the standard scenario of hybrid inflation.
} 

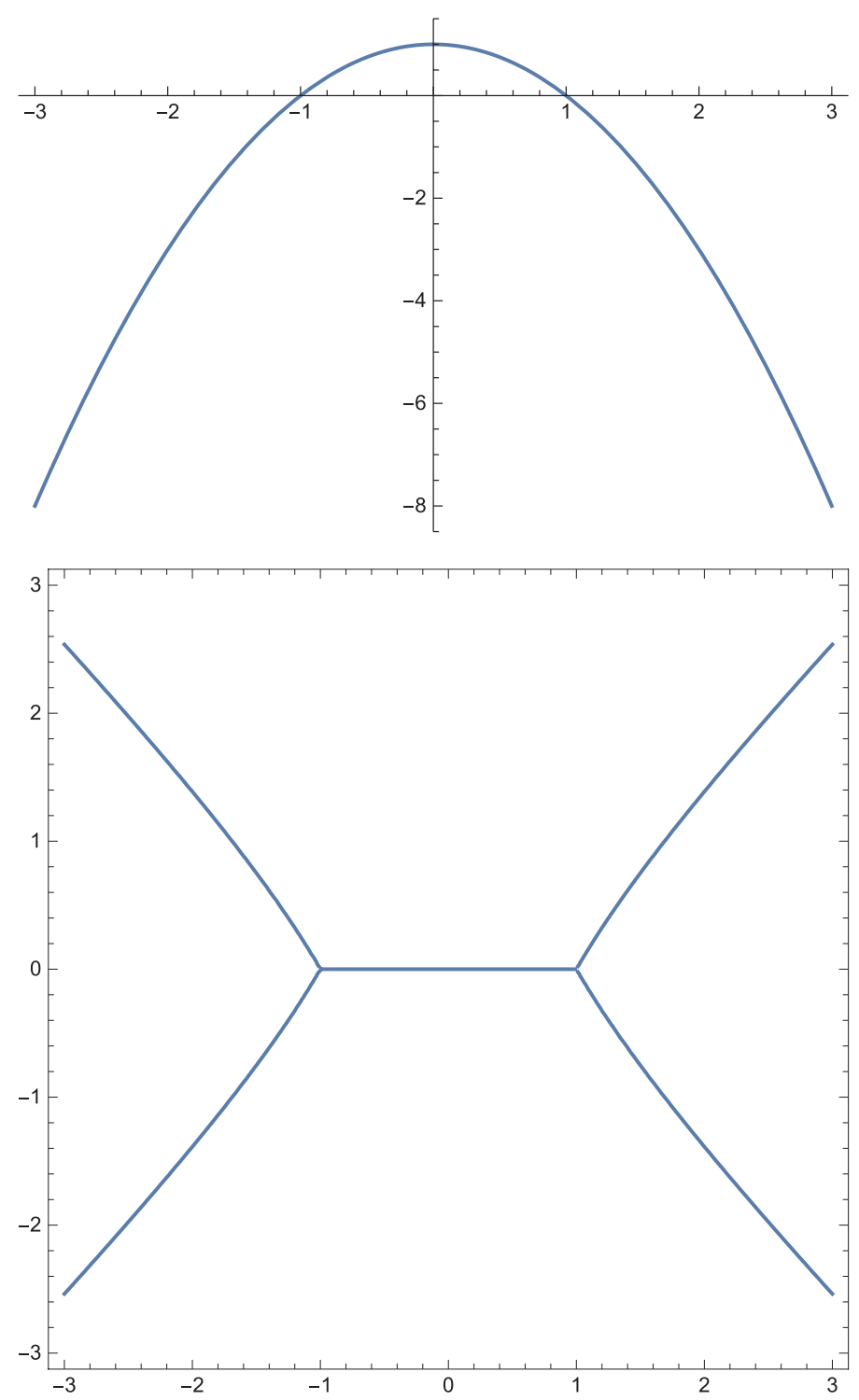

Figure 1. The potential and the Stokes lines are shown for $E<0$. In this picture, $\chi$ is tachyonic within $-1<x<1$. The classical turning points are appearing at $x= \pm 1$.

significant particle production is caused by the scattering by the classical turning point, in which $m_{\chi}^{2}<0$ is temporal. ${ }^{3}$ Therefore, although the potential and the interaction might be similar to the hybrid inflation model, we are not considering hybrid inflation. To avoid hybrid inflation, we assume $V(\chi) \ll V_{I}$, where $V_{I}$ denotes the potential during inflation, and $V(\chi)$ is the potential introduced for $\chi$.

In our case, to realize the classical turning points, the original mass $m_{0}^{2}$ has a negative sign. Then, we have $E=k^{2}-m_{0}^{2}$ for the corresponding Schrödinger equation, which realizes

\footnotetext{
${ }^{3}$ Since the particle creation may cause significant trapping of the inflaton [3, 12, 20], which may trap the inflaton within the tachyonic region after the particle production, we are focusing on the first particle production after inflation.
} 
$E<0$ during the scattering as far as $k<k_{0} \equiv m_{0}$. Our assumption $V(\chi) \ll V_{I}$ suggests that classical turning points are expected to appear near the center of the oscillation. In this case, the nonadiabatic condition is not important for the particle production, since the scattering is "classical". (Note that "classical" is used here for the classical scattering process in the scattering problem.) Using the exact solution (i.e, the Weber function), one can easily find the solution to the scattering problem. The difference from the previous solution appears in $\nu$, whose imaginary part becomes negative in this case. This flip of the sign exchanges the classical and the quantum contributions. Let us see what happens specifically. The equation of motion is

$$
\frac{d^{2} \chi}{d t^{2}}+\left[k^{2}-m_{0}^{2}+g_{2}^{2} v^{2} t^{2}\right] \chi=0
$$

where the corresponding Schrödinger equation has

$$
\begin{aligned}
E_{k} & =k^{2}-m_{0}^{2} \\
V(t) & =-\left(g_{2}^{2} v^{2}\right) t^{2} .
\end{aligned}
$$

For $k<k_{0}$, classical turning points appear at $t_{*}= \pm \frac{\sqrt{-k^{2}+m_{0}^{2}}}{g_{2} v}$ on the real axis of $t$, and $\chi$ is tachyonic within $-\frac{\sqrt{-k^{2}+m_{0}^{2}}}{g_{2} v}<t<\frac{\sqrt{-k^{2}+m_{0}^{2}}}{g_{2} v}$. Since the difference appears in $\nu$, we redefine

$$
\hat{\nu} \equiv \frac{k^{2}-m_{0}^{2}}{2 g_{2} v} i-\frac{1}{2}
$$

and

$$
\hat{\kappa} \equiv \frac{-k^{2}+m_{0}^{2}}{2 g_{2} v}>0
$$

where

$$
\hat{\nu}=-i \hat{\kappa}-\frac{1}{2}
$$

To make $\hat{\kappa}>0$, the sign of $\hat{\kappa}$ is taken to be opposite to $\kappa$. The Weber function gives

$$
\left(\begin{array}{c}
\alpha_{k}^{R} \\
\beta_{k}^{R}
\end{array}\right)=\left(\begin{array}{cc}
\sqrt{1+e^{2 \pi \hat{\kappa}}} e^{i \hat{\theta}_{1}} & i e^{\pi \hat{\kappa}+i \hat{\theta}_{2}} \\
-i e^{\pi \hat{\kappa}-i \hat{\theta}_{2}} & \sqrt{1+e^{2 \pi \hat{\kappa}}} e^{-i \hat{\theta}_{1}}
\end{array}\right)\left(\begin{array}{c}
\alpha_{k}^{L} \\
\beta_{k}^{L}
\end{array}\right),
$$

which leads to

$$
\begin{aligned}
& \left|R_{k}\right|=\frac{e^{\pi \hat{\kappa}}}{\sqrt{1+e^{2 \pi \hat{\kappa}}}}=\frac{1}{\sqrt{1+e^{-2 \pi \hat{\kappa}}}} \\
& \left|T_{k}\right|=\frac{1}{\sqrt{1+e^{2 \pi \hat{\kappa}}}}=\frac{e^{-\pi \hat{\kappa}}}{\sqrt{1+e^{-2 \pi \hat{\kappa}}}},
\end{aligned}
$$

as expected.

Now our questions are:

1. Under what conditions can we expect meaningful amplification of particle production by the classical turning points?

2. Do the conditions necessary for the amplification meet the cosmological requirements? 
Before discussing the details, to avoid confusion, we have to say that such amplification is unlikely to occur as far as the quadratic potential (i.e, the conventional $g^{2} \phi^{2} \chi^{2}$ interaction) is considered for the particle production. We are not claiming that the result is new for the community. Perhaps, this is the reason why the classical scattering has not been discussed for the cosmological preheating scenarios. In this paper, we extend the analysis to include higher terms to find a reasonable amplification by the classical scattering. The amplification appearing for the higher interaction is new.

Let us first specify the meaning of "amplification" discussed above. A typical preheating scenario considers significant particle production within the Fermi sphere $k<k_{*} \equiv$ $\sqrt{2 g_{2} v}$. Therefore, one can estimate $n \sim k_{*}^{3}$ for the scenario. If one expects "amplification" of the particle production, it is reasonable to examine whether $k_{0}>k_{*}$ is realized in the model or not. We find $k_{0}>k_{*}$ gives

$$
m_{0}^{2}>2 g_{2} v
$$

which leads to

$$
g_{2}<10^{-8}\left(\frac{m_{0}}{10^{12} \mathrm{GeV}}\right)^{2}\left(\frac{10^{16} \mathrm{GeV}}{\sqrt{v}}\right)^{2} .
$$

This alone is a simple fine-tuning of the parameters.

If one wants to avoid tachyonic $\chi$ before the onset of the inflaton oscillation, one has to consider another condition $m_{0}<g_{2} \phi_{0}$, where $\phi_{0}$ is the initial amplitude of the oscillation. Therefore, one has

$$
g_{2}^{2} \phi_{0}^{2}>m_{0}^{2}>2 g_{2} v
$$

The condition gives the lower bound given by

$$
g_{2}>10^{-4}\left(\frac{10^{18} \mathrm{GeV}}{\phi_{0}}\right)^{2}\left(\frac{\sqrt{v}}{10^{16} \mathrm{GeV}}\right)^{2} .
$$

Apparently, these conditions are contradicting. Therefore, at least in this model, the negative mass term does not cause "amplification by the classical scattering".

Similar particle production could be possible when bubbles (cosmological domain walls) collide. ${ }^{4}$ Unfortunately again, we could not find significant amplification in such a scenario.

Therefore, to find significant "amplification by the classical scattering", we are going to extend the scenario and include higher interaction.

\section{The exact WKB for preheating}

In this section, we describe the calculation based on the EWKB analysis, which gives a simple method for calculating the connection formula. Theoretically, the basic connection formulae are obtained by adding up all the WKB expansions and taking the Borel summation. The merit of using the EWKB is that once the Stokes lines are given, the connection

\footnotetext{
${ }^{4}$ For domain walls made from superpotential, the cosmological domain wall problem is not serious as far as the fine-tuning of the vacuum energy is realized by a constant term in the superpotential [21].
} 
formula is manifest for the Borel-summed WKB solutions. Note that the normalization problem is typical in the ordinary WKB method, which can be avoided by putting consistency conditions [5]. The normalization problem in the EWKB has been discussed and solved in refs. [14, 22, 23] for typical MTP (Merged pair of simple Turning Points). In this case, the factor has to be introduced on both sides of each MTP structure.

To avoid confusion, we note here that for the simple example discussed here (i.e, scattering with an inverted quadratic potential), the exact solution is already known as the Weber function and there is no obvious merit for considering alternative formulation. We are introducing the EWKB here for later convenience.

\subsection{Connection formulae by EWKB}

Typical EWKB uses $\eta \equiv \hbar^{-1} \gg 1$, instead of using the Planck constant. Following ref. [24], our starting point is the "Schrödinger equation" in quantum mechanics given by

$$
\left[-\frac{d^{2}}{d x^{2}}+\eta^{2} Q(x)\right] \psi(x, \eta)=0
$$

where

$$
Q(x)=V(x)-E
$$

for the potential $V$ and the energy $E$.

If the solution $\psi$ is written as $\psi(x, \eta)=e^{R(x, \eta)}$, we have

$$
\psi=e^{\int_{x_{0}}^{x} S(x, \eta) d x}
$$

for $S(x, \eta) \equiv \partial R / \partial x$. For $S$, we have

$$
-\left(S^{2}+\frac{\partial S}{\partial x}\right)+\eta^{2} Q=0
$$

If one expands $S$ as $S(x, \eta)=\sum_{n=-1}^{n=\infty} \eta^{-n} S_{n}$, one will find

$$
S=\eta S_{-1}(x)+S_{0}(x)+\eta^{-1} S_{1}(x)+\ldots,
$$

which leads

$$
\begin{aligned}
S_{-1}^{2} & =Q \\
2 S_{-1} S_{j} & =-\left[\sum_{k+l=j-1, k \geq 0, l \geq 0} S_{k} S_{l}+\frac{d S_{j-1}}{d x}\right](j \geq 0) .
\end{aligned}
$$

Using the relation between the odd and the even series, one will have

$$
\begin{aligned}
\psi & =\frac{1}{\sqrt{S_{\text {odd }}}} e^{\int_{x_{0}}^{x} S_{\text {odd }} d x} \\
S_{\text {odd }} & \equiv \sum_{j \geq 0} \eta^{1-2 j} S_{2 j-1} .
\end{aligned}
$$




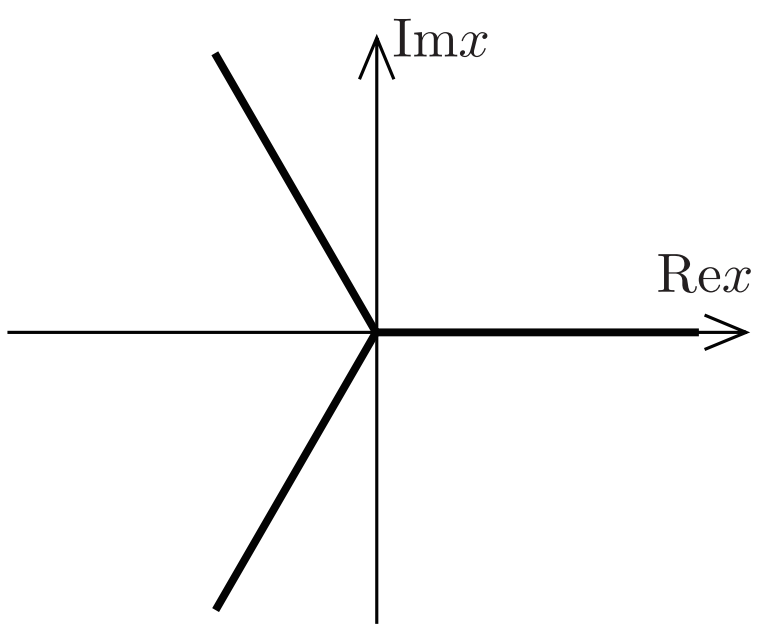

Figure 2. The Stokes lines for $Q=x$.

Depending on the sign of the first $S_{-1}= \pm \sqrt{Q(x)}$, there are two solutions $\psi_{ \pm}$, which are given by

$$
\psi_{ \pm}=\frac{1}{\sqrt{S_{\text {odd }}}} \exp \left( \pm \int_{x_{0}}^{x} S_{\text {odd }} d x\right)
$$

The above WKB expansion is usually divergent but is Borel-summable. Namely, one can consider

$$
\begin{aligned}
\psi_{ \pm} \rightarrow \Psi_{ \pm} & \equiv \int_{\mp s(x)}^{\infty} e^{-y \eta} \psi_{ \pm}^{B}(x, y) d y \\
s(x) & \equiv \int_{x_{0}}^{x} S_{-1}(x) d x
\end{aligned}
$$

where the $y$-integral is parallel to the real axis. The Stokes phenomenon in the EWKB is explained using the Airy function $(Q(x)=x)$ near the turning points. If one defines the Stokes line starting from the turning point at $x=0$ as

$$
\operatorname{Im}[s(x)]=0,
$$

The Stokes lines are the solutions of

$$
\operatorname{Im}[s(x)]=\operatorname{Im}\left[\int_{0}^{x} x^{1 / 2} d x\right]=\operatorname{Im}\left[\frac{2}{3} x^{3 / 2}\right]=0,
$$

which can be written as figure 2 . If $\operatorname{Re}[s(x)]>0, \psi_{+}$is dominant on the Stokes line, while if $\operatorname{Re}[s(x)]<0, \psi_{-}$is dominant.

The paths of integration are given in figure 3. Note that the paths overlap on the Stokes line, since the Stokes lines are defined as the solutions of $\operatorname{Im}[s(x)]=0$. Therefore, the paths may develop additional contributions when $x$ goes across the Stokes line. This is called the Stokes phenomenon. 


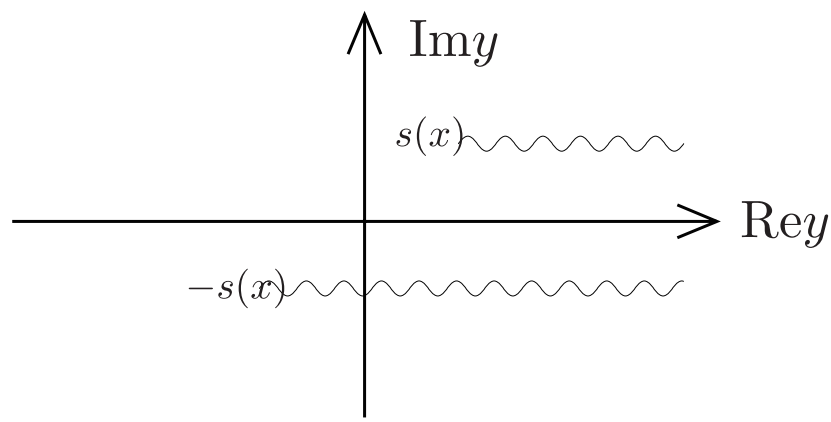

Figure 3. The wavy lines are the paths of integration in the Borel summation. The paths overlap when $\operatorname{Im}[s]=0$.

Using the above idea, one can develop the following connection formulae:

- Crossing the $\psi_{+}$-Dominant Stokes line with anticlockwise rotation (seen from the turning point)

$$
\begin{aligned}
& \Psi_{+} \rightarrow \Psi_{+}+i \Psi_{-} \\
& \Psi_{-} \rightarrow \Psi_{-}
\end{aligned}
$$

- Crossing the $\psi_{-}$-Dominant Stokes line with anticlockwise rotation (seen from the turning point)

$$
\begin{aligned}
& \Psi_{-} \rightarrow \Psi_{-}+i \Psi_{+} \\
& \Psi_{+} \rightarrow \Psi_{+}
\end{aligned}
$$

- Inverse rotation given a minus sign in front of $i$.

Let us use these simple formulae to solve the scattering problem by the inverted quadratic potential $(E>0)$. The Stokes lines are given by figure 4 , which has an MTP. The degeneracy of the Stokes line can be solved by introducing imaginary parameters such as $\eta \rightarrow \eta \pm i \eta_{\epsilon}$ or $E \rightarrow E \pm i \epsilon$. See figure 5. However, if one ignores the normalization factor, the \pm splittings are not consistent. Fortunately, in the EWKB, the factor can be calculated explicitly without relying on physical requirements [22]. For the normalization factor, we have

$$
\begin{aligned}
\psi_{ \pm} & =\frac{1}{\sqrt{S_{\text {odd }}}} \exp \left( \pm \int_{x_{0}}^{x} S_{\text {odd }} d x\right)=\psi_{ \pm}^{(\infty)} \exp \left( \pm \int_{x_{0}}^{x}\left(S_{\text {odd }}-\eta S_{-1}\right) d x\right) \\
\psi_{ \pm}^{(\infty)} & =\frac{1}{\sqrt{S_{\text {odd }}}} \exp \left( \pm \int_{x_{0}}^{x} S_{-1} d x\right) \times \exp \left( \pm \int_{\infty}^{x}\left(S_{\text {odd }}-\eta S_{-1}\right) d x\right) .
\end{aligned}
$$

Among them, what is not trivial is

$$
\exp \left( \pm \int_{x_{0}}^{x}\left(S_{\text {odd }}-\eta S_{-1}\right) d x\right)
$$




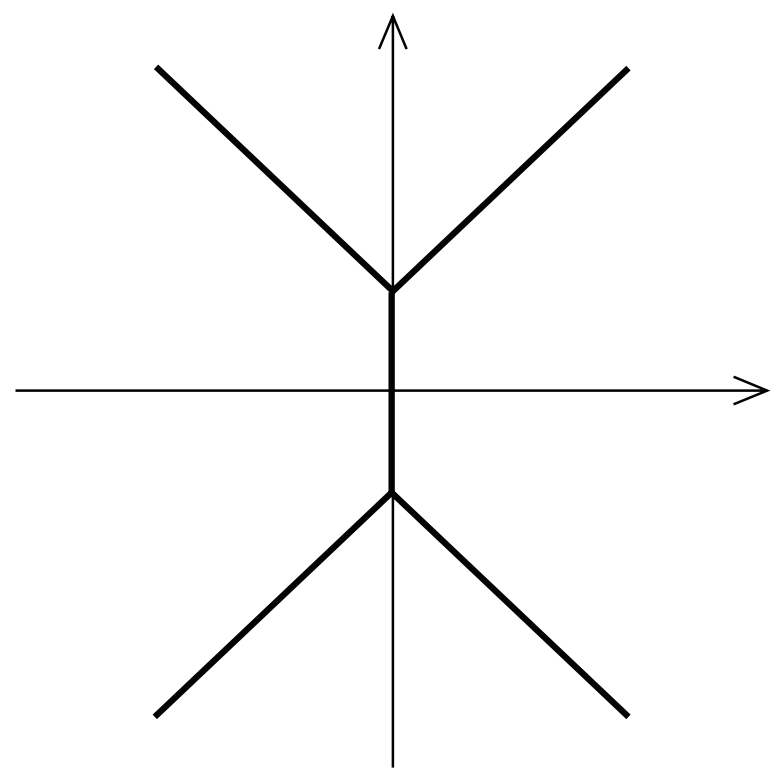

Figure 4. The Stokes lines for the inverted quadratic potential $(E>V)$. The branch points are the complex "turning points.".

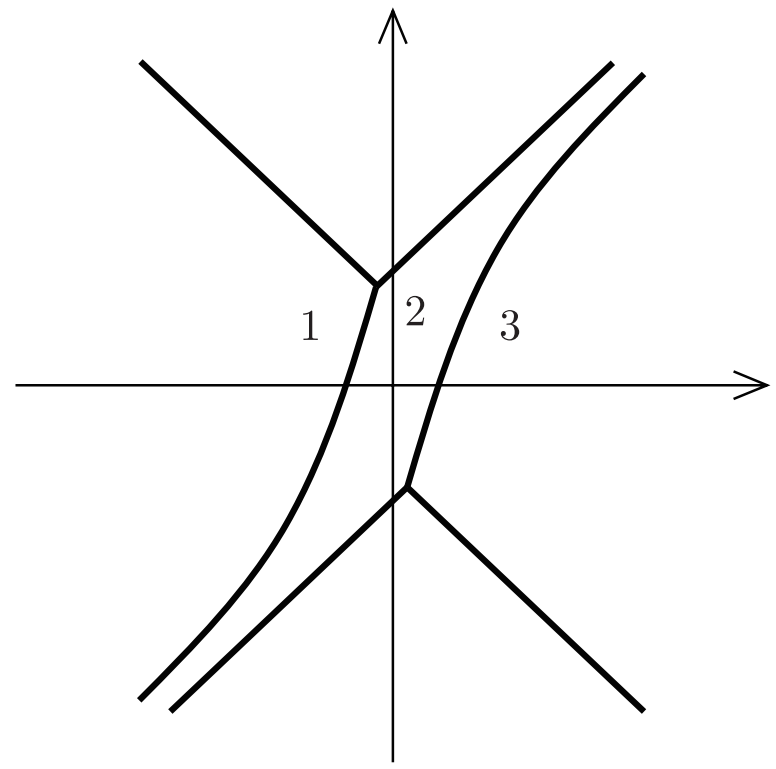

Figure 5. Split by $E \rightarrow E+i \epsilon$.

This gives for $Q(x)=\lambda-\frac{x^{2}}{4}$,

$$
2 \int_{2 \sqrt{\lambda}}^{x}\left(S_{\text {odd }}-\eta S_{-1}\right) d x=\sum_{n=1}^{\infty} \frac{2^{1-2 n}-1}{2 n(2 n-1)} B_{2 n}(i \eta \lambda)^{1-2 n}
$$

where $B_{2 n}$ is the Bernoulli number. Because of this, one has the gap given by

$$
\Psi_{+}^{(\operatorname{Im} \lambda<0)}=\sqrt{1+e^{-2 \pi \lambda \eta}} \Psi_{+}^{(\operatorname{Im} \lambda>0)} .
$$


The calculation can be generalized to give the factor appearing on both sides of more generic MTP [23]. In this paper, based on these mathematical results, we derive the MTP factor from the simple consistency relation.

Analytic continuation of the integration requires identification of the sheet on which the path is placed. Since a cut always appears from a turning point, the sign of the integration will be inverted when the path goes around a turning point. Therefore, if the integration path going back and forth to the turning point makes a turn around the turning point, the integration does not cancel out. This property is often used to define the integration in the EWKB.

\subsection{Exact WKB for $E>0$ inverted quadratic potential}

Now we can calculate the connection formula for the inverted quadratic potential using the EWKB. We start with

$$
\frac{d^{2} \psi}{d t^{2}}+\left[k^{2}+g_{2}^{2} v^{2} t^{2}\right] \psi=0
$$

The initial time $t$ starts from the region 1 in figure 5 and it connects to the right region 3 through the region 2. First, we consider the WKB solution whose integral starts from a turning point $t=i \frac{k}{g_{2} v} \equiv t_{*}^{+}$. This point can be shared both in region 1 and 2 . We are crossing the $\Psi_{-}$dominant Stokes line anticlockwise, and the connection formula becomes

$$
\left(\begin{array}{l}
\Psi_{+}^{(2 u)} \\
\Psi_{+}^{(2 u)}
\end{array}\right)=\left(\begin{array}{ll}
1 & 0 \\
i & 1
\end{array}\right)\left(\begin{array}{l}
\Psi_{+}^{(1 u)} \\
\Psi_{+}^{(1 u)}
\end{array}\right) .
$$

Here, $(1 u)$ means "in the region 1 , integration starts from the upper turning point at $t=t_{*}^{+}$". The starting point of the integration has to be changed when one moves from the region 2 to 3 . We define

$$
K_{u d} \equiv \int_{t_{*}^{+}}^{t_{*}^{-}} S_{\text {odd }} d t
$$

where $t_{*}^{-} \equiv-i \frac{k}{g_{2} v}$ and

$$
\left(\begin{array}{c}
\Psi_{+}^{(2 u)} \\
\Psi_{+}^{(2 u)}
\end{array}\right) \rightarrow\left(\begin{array}{cc}
e^{K_{u d}} & 0 \\
0 & e^{-K_{u d}}
\end{array}\right)\left(\begin{array}{c}
\Psi_{+}^{(2 d)} \\
\Psi_{+}^{(2 d)}
\end{array}\right) .
$$

Now the path crosses the $\Psi_{+}$dominant Stokes line clockwise, which gives the connection formula

$$
\left(\begin{array}{l}
\Psi_{+}^{(3 d)} \\
\Psi_{+}^{(3 d)}
\end{array}\right)=\left(\begin{array}{cc}
1 & -i \\
0 & 1
\end{array}\right)\left(\begin{array}{l}
\Psi_{+}^{(2 d)} \\
\Psi_{+}^{(2 d)}
\end{array}\right) .
$$

Let us derive the normalization factor using a simple consistency relation. For the normalization factor $N$, we have [22]

$$
\begin{aligned}
& \Psi_{ \pm}^{1 \infty}=N^{\mp} \Psi_{ \pm}^{(1+)} \\
& \Psi_{ \pm}^{3 \infty}=N^{\mp} \Psi_{ \pm}^{(3-)}
\end{aligned}
$$


which gives the connection formula from 1 to 3 as

$$
\left(\begin{array}{c}
\Psi_{+}^{(3 \infty)} \\
\Psi_{+}^{(3 \infty)}
\end{array}\right)=U_{N} U_{+c} U_{u d} U_{-a} U_{N}\left(\begin{array}{c}
\Psi_{+}^{(1 \infty)} \\
\Psi_{+}^{(1 \infty)}
\end{array}\right)
$$

where

$$
\begin{aligned}
U_{+a} & \equiv\left(\begin{array}{ll}
1 & i \\
0 & 1
\end{array}\right) \\
U_{+c} & \equiv\left(\begin{array}{cc}
1 & -i \\
0 & 1
\end{array}\right) \\
U_{-a} & \equiv\left(\begin{array}{ll}
1 & 0 \\
i & 1
\end{array}\right) \\
U_{-c} & \equiv\left(\begin{array}{cc}
1 & 0 \\
-i & 1
\end{array}\right) \\
U_{u d} & \equiv\left(\begin{array}{cc}
e^{K_{u d}} & 0 \\
0 & e^{-K_{a d}}
\end{array}\right) \\
U_{N} & \equiv\left(\begin{array}{cc}
N & 0 \\
0 & N^{-1}
\end{array}\right) .
\end{aligned}
$$

The result is

$$
\left(\begin{array}{cc}
N^{2}\left(e^{-K_{u d}}+e^{K_{u d}}\right. & -i e^{-K_{u d}} \\
i e^{-K_{u d}} & \frac{e^{-K_{u d}}}{N^{2}}
\end{array}\right) .
$$

To make the diagonal elements consistent, we have

$$
|N|^{-2}=\sqrt{1+e^{2 K_{u d}}} .
$$

Finally, the connection matrix becomes

$$
\left(\begin{array}{cc}
\sqrt{1+e^{-2 K_{u d}}} & -i e^{-K_{u d}} \\
i e^{-K_{u d}} & \sqrt{1+e^{-2 K_{u d}}}
\end{array}\right),
$$

where the phase of $N$, which is calculable in the EWKB but not calculable from the simple consistency relation only, has been neglected for simplicity.

The same calculation is possible for $E<0$, in which classical turning points appear. For later convenience, we explicitly show the Stokes lines in figure 6. The connection matrix for crossing the cut is

$$
\begin{aligned}
U_{c c} & \equiv\left(\begin{array}{ll}
0 & i \\
i & 0
\end{array}\right) \\
U_{c a} & \equiv\left(\begin{array}{cc}
0 & -i \\
-i & 0
\end{array}\right),
\end{aligned}
$$




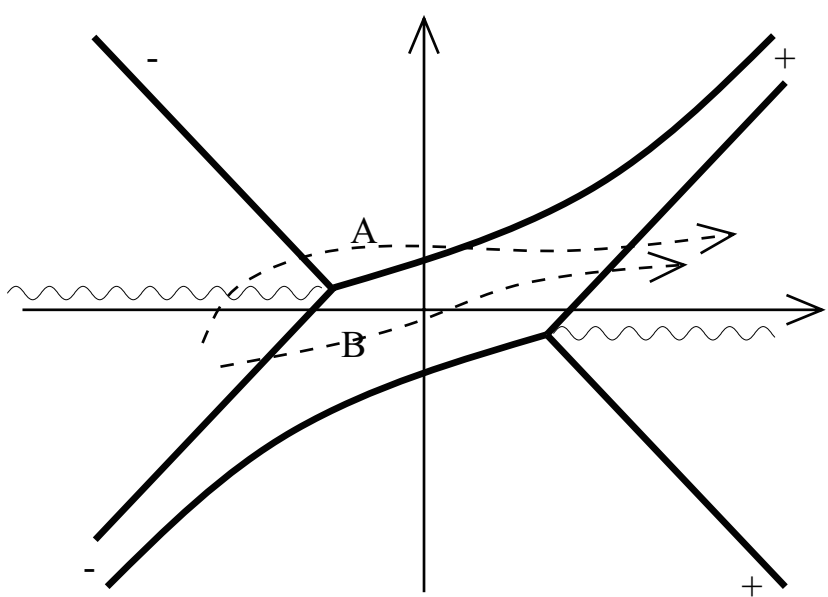

Figure 6. The Stokes lines for the inverted quadratic potential with $E<0$. The wavy lines are the cuts.

where $c c$ and $c a$ denote the clockwise and the anticlockwise motion across the cut. For the path $\mathrm{A}$, the connection matrix is

$$
U_{N} U_{+c} U_{L R} U_{+c} U_{-c} U_{c c} U_{N}=\left(\begin{array}{cc}
\left(e^{-K_{L R}}+e^{K_{L R}}\right. & N^{2} \\
i e^{-K_{L R}} & \frac{e^{-K_{L R}}}{N^{2}}
\end{array}\right)
$$

where

$$
U_{L R} \equiv\left(\begin{array}{ll}
e^{K_{L R}} & \\
& e^{-K_{L R}}
\end{array}\right)
$$

and

$$
K_{L R} \equiv \int_{t_{*}^{L}}^{t_{*}^{R}} S_{\text {odd }} d t, \quad t_{*}^{L} \equiv-\frac{k}{g_{2} v}, \quad t_{*}^{R} \equiv \frac{k}{g_{2} v}
$$

Again, the consistency relation gives

$$
|N|^{-2}=\sqrt{1+e^{2 K_{L R}}}
$$

The result is

$$
\left(\begin{array}{cc}
\sqrt{1+e^{-2 K_{L R}}} & -i e^{-K_{L R}} \\
i e^{-K_{L R}} & \sqrt{1+e^{-2 K_{L R}}}
\end{array}\right)
$$

For the path B, one has

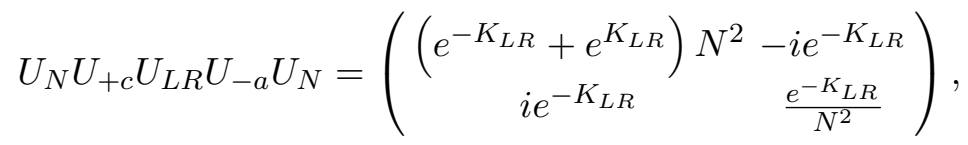

which is giving the identical result with the path $\mathrm{A}$.

Now we can calculate the connection matrix for more exotic potential using the simple EWKB. 


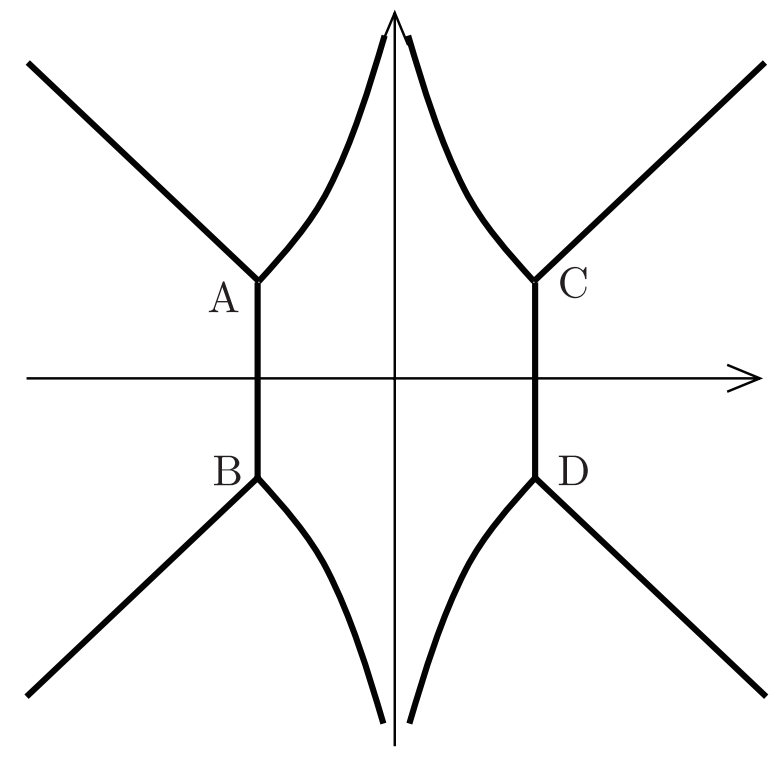

Figure 7. The Stokes lines for the inverted quartic potential with $E>0$.

\section{EWKB for higher dimensional interaction}

\subsection{Quantum scattering for inverted quartic potential}

We start with the inverted quartic potential $Q(x)=E+x^{4}$, which has four (complex) turning points

$$
x_{n}=E^{\frac{1}{4}} e^{i \frac{(2 n-1) \pi}{4}}, \quad(n=1, \ldots 4) .
$$

The Stokes lines are shown in figure 7. The previous calculation of the inverted quadratic potential with $E>0$ is suggesting that the connection matrix in this case is given by the product of two matrices, each of which (including the MTP factors) has to be calculated for each MTP. Neglecting the phase parameters, the significant difference from the conventional quadratic potential may only appear in the integration factors, which are connecting the turning points $A-B$ and $C-D$.

To make a more specific calculation, we consider the Lagrangian given by

$$
\mathcal{L}_{\chi}=\frac{1}{2} \partial_{\mu} \chi \partial^{\nu} \chi-\frac{1}{2} m_{0}^{2} \chi^{2}-\frac{1}{2} g_{2}^{2} \phi(t)^{2} \chi^{2}-g_{4}^{2} \frac{\phi(t)^{4}}{M_{*}^{2}} \chi^{2},
$$

where $M_{*}$ denotes the cut-off scale of the effective action. One may take $M_{*}$ as large as the GUT(Grand Unified Theory) scale or the Planck scale. The effective mass of $\chi$ is given by

$$
m_{\chi}^{2}(t)=m_{0}^{2}+g_{2}^{2} \phi(t)^{2}+g_{4}^{2} \frac{\phi(t)^{4}}{M_{*}^{2}} .
$$

The equation of motion is

$$
\frac{d^{2} \chi}{d t^{2}}+\left[k^{2}+m_{\chi}^{2}(t)\right] \chi=0
$$


Again, we assume that the approximation $\phi(t) \simeq v t$ is possible when the particles are produced. Then, we have the Schrödinger equation for the scattering problem with the inverted quartic potential, which is given by

$$
\frac{d^{2} \chi}{d t^{2}}+\left[k^{2}+m_{0}^{2}+g_{2}^{2} v^{2} t^{2}+\frac{g_{4}^{2} v^{4}}{M_{*}^{2}} t^{4}\right] \chi=0 .
$$

Here the "energy" and the "potential" are given by

$$
\begin{aligned}
E_{k} & =k^{2}+m_{0}^{2} \\
V(t) & =-\left(g_{2}^{2} v^{2}\right) t^{2}-\frac{g_{4}^{2} v^{4}}{M_{*}^{2}} t^{4} .
\end{aligned}
$$

According to the calculation we have given for the quadratic potential, we translate

$$
\begin{aligned}
z & =\left(\frac{g_{4}^{2} v^{4}}{M_{*}^{2}}\right)^{\frac{1}{6}} t \\
a_{4}^{2} & \equiv\left(\frac{g_{4}^{2} v^{4}}{M_{*}^{2}}\right)^{\frac{1}{3}}=v\left(\frac{g_{4}^{2} v}{M_{*}^{2}}\right)^{\frac{1}{3}}
\end{aligned}
$$

to make these quantities dimensionless, we have

$$
\begin{gathered}
V(z)=-z^{4}-g_{2}^{2}\left(\frac{M_{*}^{2}}{g_{4}^{2} v}\right)^{\frac{2}{3}} \\
E_{k}=\frac{k^{2}+m_{0}^{2}}{a_{4}^{2}} \equiv \kappa_{4}^{2} .
\end{gathered}
$$

Defining $\delta_{4} \equiv\left(\frac{g_{4}^{2} v}{M_{*}^{2}}\right)^{\frac{1}{3}} \ll 1$, we have

$$
\begin{aligned}
V(z) & =-z^{4}-\frac{g_{2}^{2}}{\delta_{4}^{2}} z^{2} \\
E_{k} & =\kappa_{4}^{2}=\frac{k^{2}+m_{0}^{2}}{v \delta_{4}} .
\end{aligned}
$$

We start with the calculation for $g_{2}=0$ (pure quartic). One can find a more explicit calculation in ref. [14], in which the Stokes lines are shown to split into the form given in figure 8. Here, what we want is not the exact formula but an estimation of the elements of the connection matrix. In this case, the parameter which determines the magnitude of particle creation is the integral connecting MTP, which is shown in figure 9. For $Q(x)=$ $V(x)-E_{k}=-x^{4}-\kappa_{4}^{2}$, the integration factor $K_{A B}$ connecting the turning points at $\left(t_{A}=\sqrt{\kappa_{4}} e^{i \frac{3 \pi}{4}}\right)$ and $\left(t_{B}=\sqrt{\kappa_{4}} e^{i \frac{5 \pi}{4}}\right)$ becomes

$$
\begin{aligned}
K_{A B} & =2 \int_{A}^{B} \sqrt{-x^{4}-\kappa_{4}^{2}} d x \\
& \simeq 1.2 \kappa_{4}^{\frac{3}{2}} .
\end{aligned}
$$




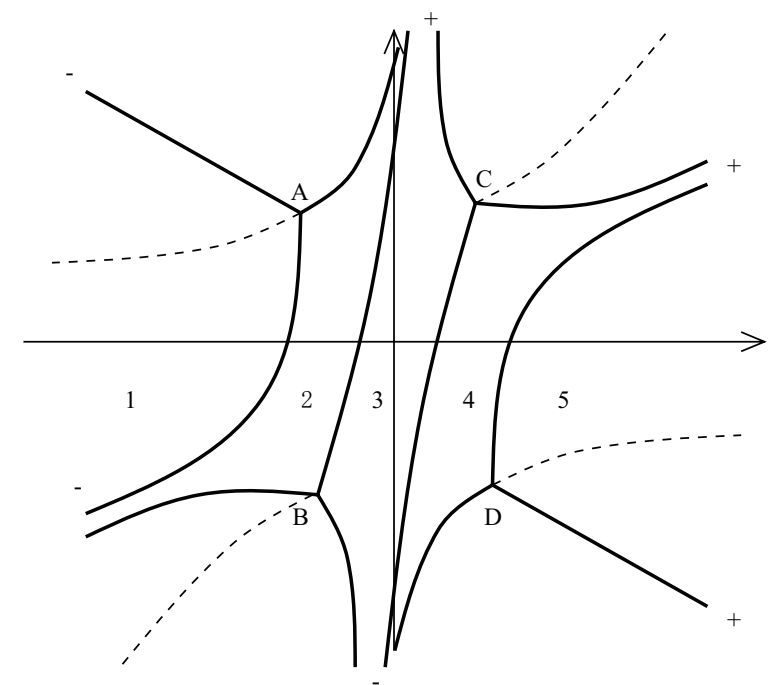

Figure 8. Split Stokes lines of MTPs in the simple quartic potential.

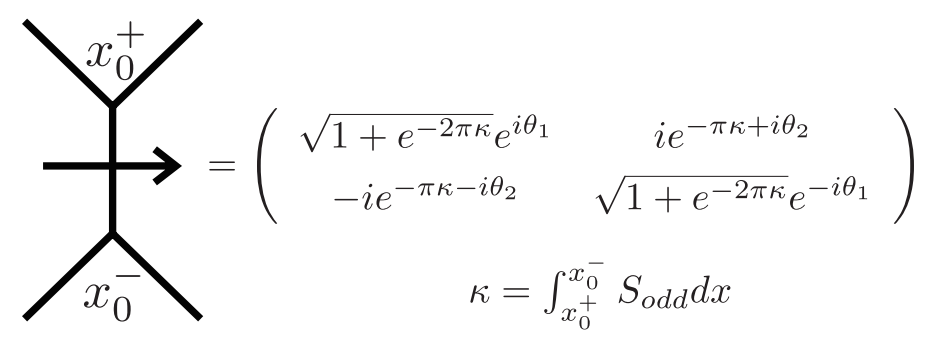

Figure 9. An MTP structure and its integration factor.

If we define the critical parameter $k_{*}$ by

$$
1.2 \kappa_{4}^{\frac{3}{2}} \simeq 1
$$

we have

$$
k_{*} \simeq \sqrt{v} \times\left(\frac{\delta_{4}}{1.3}\right)^{1 / 2}
$$

in a case of $k_{*} \gtrsim m_{0}$. Typically, we have

$$
\delta_{4} \simeq 10^{-5 / 3}\left(\frac{g_{4}}{0.1}\right)^{1 / 3}\left(\frac{\sqrt{v}}{10^{16} \mathrm{GeV}}\right)^{2 / 3}\left(\frac{10^{18} \mathrm{GeV}}{M_{*}}\right)^{2 / 3}
$$

which gives

$$
k_{*} \sim 0.1 \sqrt{v}
$$

This result shows that despite the significant suppression by the cut-off scale appearing in the higher interaction, the integration factor may not introduce significant suppression to the particle production. From this result, one can see that the typical (explicit) interaction is not always necessary for the cosmological preheating scenario, since higher (Planck-suppressed) terms can cause significant particle production after inflation. This result is consistent with our earlier works $[12,13]$. Note also that the result is obtained 


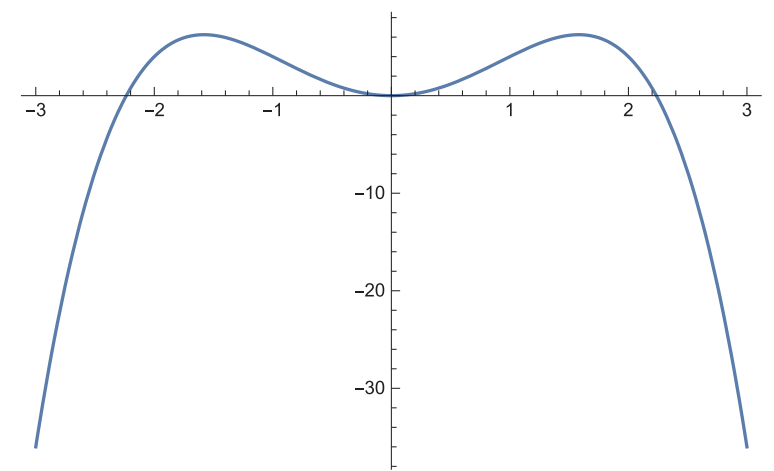

Figure 10. Inverted double-well potential of $V=5 x^{2}-x^{4}$.

for a single process, which means that parametric resonance is not assumed to obtain significant particle production by the Planck-suppressed interaction. This result may affect the conventional estimation of cosmological moduli production, since any (hidden) particle can have Planck-suppressed interaction.

\subsection{Classical scattering for inverted double-well potential}

Previously we analyzed the cosmological effect of classical turning points of the inverted quadratic potential and mentioned the "amplification", which had specific meanings. Here we consider amplification by the classical turning points of the higher interaction.

To introduce scattering by a classical turning point, we consider a negative coefficient for the quadratic term (i.e., $g_{2}^{2}$ is replaced by $-g_{2}^{2}$ ). Just for simplicity, we put $m_{0}=0$. Typically, the potential becomes an inverted double-well form, which is shown in figure 10 . We consider the scattering problem of the Schrödinger equation with

$$
\begin{gathered}
V(z)=-z^{4}+\frac{g_{2}^{2}}{\delta_{4}^{2}} z^{2} \\
E_{k}=\kappa_{4}^{2}=\frac{k^{2}}{v \delta_{4}} .
\end{gathered}
$$

The two bumps of the potential are placed at

$$
z= \pm \frac{g_{2}}{\sqrt{2} \delta_{4}}
$$

and the height is given by

$$
V\left( \pm \frac{g_{2}}{\sqrt{2} \delta_{4}}\right)=\frac{1}{4} \frac{g_{2}^{4}}{\delta_{4}^{4}}
$$

Therefore, the "tachyonic" region appears when $E_{k}<V(z)$. If we define $k_{0}$ by

$$
\frac{k_{0}^{2}}{v \delta_{4}}=\frac{1}{4} \frac{g_{2}^{4}}{\delta_{4}^{4}},
$$

we find

$$
k_{0}=\frac{1}{2} v \frac{g_{2}^{2}}{\delta_{4}^{3 / 2}} \simeq \sqrt{v} \times 10^{3 / 2} \times\left(\frac{g_{2}}{10^{-2}}\right)^{2}\left(\frac{10^{-11 / 3}}{\delta_{4}}\right)^{\frac{3}{2}}
$$


From the above result, one can confirm in this case that there can be the "amplification" of the particle production by the classical turning points. This is our new result in this paper.

\section{Fermions}

Typically, equation of motion of a fermion is described by a pair of first-order differential equations. We are going to see how higher terms appear in a fermionic model and discuss its cosmological implications. Note that for fermions, higher (quartic) potential may appear in the corresponding Schrödinger equation without introducing higher dimensional interaction to the theory. We are going to discuss the above last topic first.

\subsection{Landau-Zener model and kinematic particle creation in cosmology}

We first review the Landau-Zener model and explain its relation between cosmological particle creation. We start with a pair of first-order differential equations below

$$
i \hbar \frac{d}{d t}\left(\begin{array}{l}
\psi_{1} \\
\psi_{2}
\end{array}\right)=\left(\begin{array}{cc}
-\frac{v}{2} t & \Delta \\
\Delta & +\frac{v}{2} t
\end{array}\right)\left(\begin{array}{l}
\psi_{1} \\
\psi_{2}
\end{array}\right)
$$

where $v>0$ and $\Delta$ is real. Decoupling the equations, we have

$$
\begin{aligned}
& {\left[\frac{d^{2}}{d t^{2}}+\frac{1}{\hbar^{2}}\left(\Delta^{2}+\frac{1}{4} v^{2} t^{2}\right)-\frac{i}{\hbar} \frac{v}{2}\right] \psi_{1}=0} \\
& {\left[\frac{d^{2}}{d t^{2}}+\frac{1}{\hbar^{2}}\left(\Delta^{2}+\frac{1}{4} v^{2} t^{2}\right)+\frac{i}{\hbar} \frac{v}{2}\right] \psi_{2}=0 .}
\end{aligned}
$$

These are the Schrödinger equations with $V=-\frac{1}{\hbar^{2}} \cdot \frac{1}{4} v^{2} t^{2}$ and $E=\frac{1}{\hbar^{2}} \cdot \Delta^{2} \pm \frac{i}{\hbar} \frac{v}{2}$, where the energy is shifted by the imaginary part, which has an extra $\hbar$. Therefore, we have to consider a generalized $Q(z, \eta)$ for the EWKB analysis. The EWKB analysis of $Q(z, \eta)$ has been discussed in ref. [25], in which it has been suggested that both the turning points and the Stokes lines have to be calculated for the leading terms if one expands $Q(z, \eta)$ with $\eta^{-n}$. Hence, we analyse the Stokes lines by

$$
\left[\frac{d^{2}}{d t^{2}}+\Delta^{2}+\frac{1}{4} v^{2} t^{2}\right] \psi=0
$$

where the imaginary part has been removed and $\hbar=1$ is chosen. See also figure 11.

Let us first calculate the connection matrix using the exact solution based on the Weber function. Defining $z=i \sqrt{v} e^{i \pi / 4} t\left(z^{2}=-i v t^{2}\right)$, we have

$$
\left[\frac{d^{2}}{d z^{2}}+\left(n+\frac{1}{2}-\frac{1}{4} z^{2}\right)\right] \psi_{1}(z)=0 .
$$

Here we defined

$$
n \equiv i \frac{\Delta^{2}}{v}
$$

This is nothing but the standard equation of the Weber function. The solutions are given by a pair of independent functions among $D_{n}(z), D_{n}(-z), D_{-n-1}(i z), D_{-n-1}(-i z)$. According 


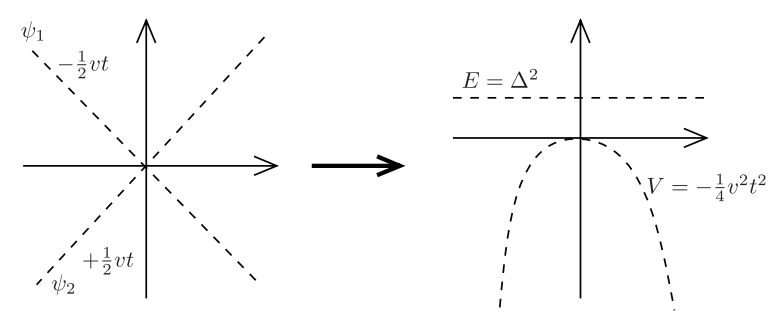

Figure 11. Left: state crossing in the Landau-Zener model. Right: after decoupling the equations, the state crossing of the Landau-Zener model corresponds to the scattering problem of the Schrödinger equation.

to the previous calculations, we can easily find the connection matrix connecting the initial $\left(\psi^{i}\right)$ to the end $\left(\psi^{e}\right)$ states.

$$
\left(\begin{array}{l}
\psi_{1}^{e} \\
\psi_{2}^{e}
\end{array}\right)=\left(\begin{array}{cc}
e^{-\pi \kappa} e^{i \theta_{1}} & -\sqrt{1-e^{-2 \pi \kappa}} e^{i \theta_{2}} \\
\sqrt{1-e^{-2 \pi \kappa}} e^{-i \theta_{2}} & e^{-\pi \kappa} e^{-i \theta_{1}}
\end{array}\right)\left(\begin{array}{l}
\psi_{1}^{i} \\
\psi_{2}^{i}
\end{array}\right),
$$

where

$$
\kappa \equiv \frac{\Delta^{2}}{v}
$$

Note however that $\psi_{1,2}$ are not the adiabatic states. Therefore, one can see that the "translation" becomes significant when $v \rightarrow 0$. To avoid confusion, the above matrix has to be given for the adiabatic states, which are not identical to $\psi_{1,2} \cdot{ }^{5}$ We thus define the adiabatic states to have the matrix given by

$$
\left(\begin{array}{l}
\Psi_{1}^{+} \\
\Psi_{2}^{+}
\end{array}\right)=\left(\begin{array}{cc}
\sqrt{1-e^{-2 \pi \kappa}} & e^{-\pi \kappa} \\
e^{-\pi \kappa} & -\sqrt{1-e^{-2 \pi \kappa}}
\end{array}\right)\left(\begin{array}{l}
\Psi_{1}^{-} \\
\Psi_{2}^{-}
\end{array}\right),
$$

where phase parameters are neglected.

Seeing the relationship between the Landau-Zener model and the scattering for the Schrödinger equation, one will find that the original (linear) $D_{1} \equiv-v t, D_{2} \equiv+v t$ in the diagonal elements are giving the quadratic potential $-\frac{1}{4} v^{2} t^{2}$ for the Schrödinger equation. Finally, the problem is equivalent to the scattering with $E=\Delta^{2}$ and $V(t) \equiv-\frac{1}{4} v^{2} t^{2}$. (See also figure 11.) The discussion of the EWKB suggests that the imaginary part of $E$, which is the next order to $\Delta^{2}$, does not change the connection matrix because it does not change the Stokes lines.

Since cosmological particle production deals with oscillatory phenomena, which means that the diagonal elemants of the Landau-Zener model is an approximation of a sinusoidal function, the intersection of the two states may not be approximated by $\pm v t$. To explain the situation, we prepared figure 12. As is shown in figure 12, two states may not cross but just approach for a short time. In this case, the diagonal elements are not approximated by $D_{1}=-D_{2}=-v t / 2$ but by $D_{1}=-D_{2}=-\epsilon-a t^{2}$. Then, one has to calculate the transition when the velocity vanishes at $t=0$. Since the velocity vanishes, one cannot use the original argument. We are going to solve this problem by using the EWKB. As is

\footnotetext{
${ }^{5}$ Note that usually the "adiabatic energy" is given by $E_{ \pm}= \pm \sqrt{\Delta^{2}+v^{2} t^{2} / 4}$, which do not intersect.
} 


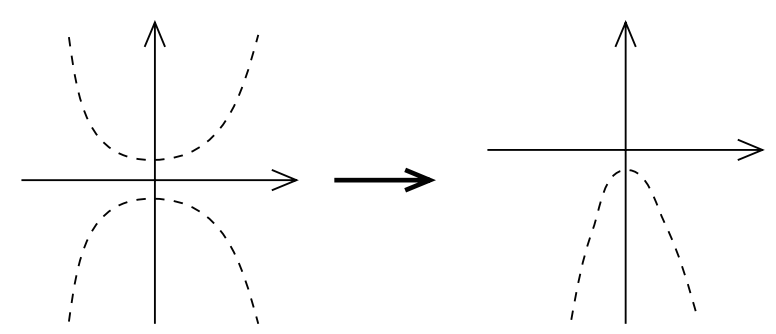

Figure 12. Left: states in the Landau-Zener model, when two states do not cross. Right: the situation is shown for the decoupled (Schrödinger) equation. The left is simply showing the diagonal elements, not the adiabatic energy.

shown in figure 12 , states (the original states $\psi_{1,2}$ ) are not crossing but approach to the distance $2 \epsilon$, and then moves away from each other.

In this case, in contrast to the conventional Landau-Zener model, one can identify the original states as the adiabatic states, since there is no crossing of the states. (i.e, we do not have to consider $\psi_{1,2} \rightarrow \Psi_{1,2}$.)

We start with the equation given by

$$
i \frac{d}{d t}\left(\begin{array}{l}
\psi_{1} \\
\psi_{2}
\end{array}\right)=\left(\begin{array}{cc}
-\left(a t^{2}+\epsilon\right) & \Delta \\
\Delta & a t^{2}+\epsilon
\end{array}\right)\left(\begin{array}{l}
\psi_{1} \\
\psi_{2}
\end{array}\right) .
$$

Combining the equations, we find

$$
\left[\frac{d^{2}}{d t^{2}}+\left(\Delta^{2}-i(2 a t)\right)+\left(a t^{2}+\epsilon\right)^{2}\right] \psi_{1}=0
$$

Again, the imaginary part should have an additional $\hbar$ and can be neglected, since our focus is the connection matrix calculated from the Stokes lines. We thus have

$$
\left[\frac{d^{2}}{d t^{2}}+\Delta^{2}+\left(a t^{2}+\epsilon\right)^{2}\right] \psi=0 .
$$

for the calculation. This equation is nothing but the Schorödinger equation for the scattering problem with the inverted quartic potential

$$
V(t)=-\left(a t^{2}+\epsilon\right)^{2}-\Delta^{2}<0
$$

and the energy $E=0$. Now the connection matrix can be calculated along the real axis, as we have shown for the bosonic preheating scenario with the higher intersection. For the EWKB, we have

$$
Q(t)=-\Delta^{2}-\epsilon^{2}-a^{2} t^{4}-2 a \epsilon t^{2} .
$$

The stokes lines for eq. (4.12) is given by figure 13, which shows that the connection matrix is calculated from the two MTPs. Again, the important factor that determines the particle production is the integral with respect to the MTPs.

We are going to examine these results from the viewpoint of cosmological particle production and discuss the physics behind them. 


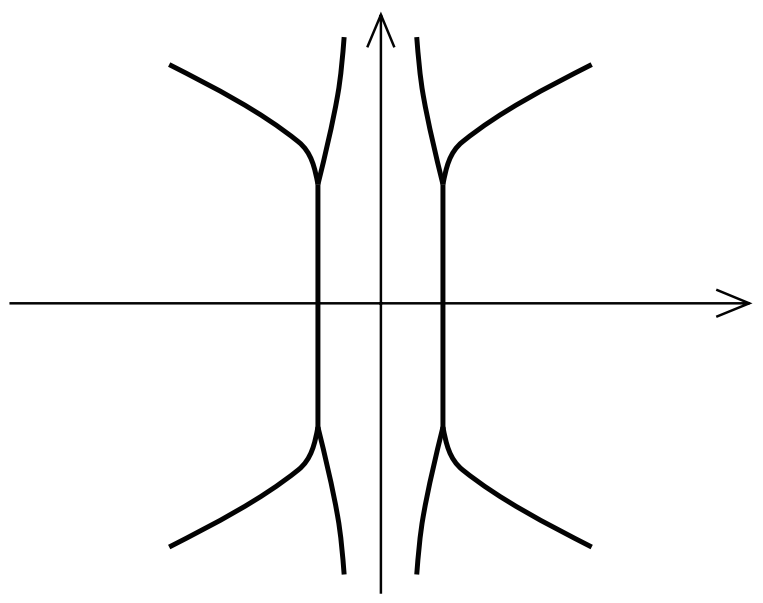

Figure 13. The Stokes lines for the fermion preheating when "crossing" is not significant.

\subsection{Cosmological particle production of fermions}

Since the fermionic preheating with a linear $\phi(t)$ is an old idea, we carefully follow ref. [26] to avoid confusion. We consider a Dirac fermion whose mass is given by

$$
m_{D}(t)=m_{0}+g \phi(t)
$$

where $m_{D}$ is assumed to be real. The Dirac equation is given by

$$
\left(i \not \partial-m_{D}\right) \psi=0,
$$

whose solution can be decomposed as

$$
\psi=\int \frac{d^{3} k}{(2 \pi)^{3}} e^{-i \boldsymbol{k} \cdot \boldsymbol{x}} \sum_{s}\left[u_{\boldsymbol{k}}^{s}(t) a_{\boldsymbol{k}}^{s}+v_{\boldsymbol{k}}^{s}(t) b_{-\boldsymbol{k}}^{s \dagger}\right] .
$$

Choosing the momentum along the third direction $k=k_{z}$, and introducing $u_{ \pm}$following ref. [26], one can obtain a two-component differential equation given by ${ }^{6}$

$$
\dot{u}_{ \pm}=i k u_{\mp} \mp i m_{D} u_{ \pm} .
$$

The equation can be written in the following form

$$
i \frac{d}{d t}\left(\begin{array}{l}
u_{+} \\
u_{-}
\end{array}\right)=\left(\begin{array}{cc}
m_{0}+g \phi(t) & -k \\
-k & -m_{0}-g \phi(t)
\end{array}\right)\left(\begin{array}{l}
u_{+} \\
u_{-}
\end{array}\right),
$$

which is giving the starting point of the Landau-Zener model. Combining the equations, we have [26]

$$
\ddot{u}_{ \pm}+\left[\omega^{2} \pm i \dot{m}_{D}\right] u_{ \pm}=0,
$$

\footnotetext{
${ }^{6}$ According to [26], the representation of the gamma matrices are chozen as

$$
\gamma^{0}=\left(\begin{array}{cc}
\mathbf{1} & \\
& -\mathbf{1}
\end{array}\right), \quad \gamma^{1}=\left(\begin{array}{c}
-i \sigma^{2} \\
-i \sigma^{2}
\end{array}\right), \quad \gamma^{2}=\left(\begin{array}{c}
i \sigma^{1} \\
i \sigma^{1}
\end{array}\right), \quad \gamma^{3}=\left(\begin{array}{c}
\mathbf{1} \\
-\mathbf{1}
\end{array}\right) .
$$
}


where we defined $\omega(t)^{2}=k^{2}+m_{D}^{2}$. In writing the Stokes lines, the imaginary term $i \dot{m}_{D}$ has to be neglected, since it has an additional $\hbar$. The typical preheating scenario considers significant particle creation near the Enhanced Symmetric Point(ESP), where one expects $m_{D}=0$ and $v \neq 0$. In this case, the motion during particle production is given by $\phi(t) \simeq v t$, which leads to the state crossing. Of course, this expectation is true when $m_{0}$ is small. Even for large $m_{0}$, fermionic preheating expects $m_{D}=0$ when $\phi\left(t_{0}\right)=-\frac{m_{0}}{g}$, but on such point the linear approximation may not be a good approximation if $\phi\left(t_{0}\right)$ appears near the edge of the oscillation, or even does not appear, as is already shown in figure 12.

Now we can examine "the Landau-Zener model without the state crossing" from the cosmological viewpoint. If $m_{0}$ is large and the mass is given by $m_{D}(t)=M(1+$ $\left.\Gamma(t) \cos m_{\phi} t\right)$, where $\Gamma(t)$ is introduced to describe the damping of the oscillation, the particle creation may be significant when the above (linear) approximation is no longer valid. This may happen during the first oscillation. In that case, one has to calculate the particle production with

$$
m_{D} \simeq \frac{M \Gamma m_{\phi}^{2}}{2}\left(t-t_{0}\right)^{2}+M(1-\Gamma) .
$$

Assuming $\Gamma \simeq 1$ for simplicity, the corresponding Schrödinger equation has

$$
\begin{aligned}
E & =k^{2} \\
V(t) & =-\frac{M^{2} m_{0}^{4}}{4}\left(t-t_{0}\right)^{4} \mp i M m_{0}^{2}\left(t-t_{0}\right) .
\end{aligned}
$$

Again, the particle production can be calculated in terms of the scattering problem of the Schrödinger equation, but in this case the quadratic potential is replaced by the quartic potential. This means that the production of heavy fermions (such as right-handed Majorana fermions or GUT particles decaying to generate baryon/lepton number) could be affected by such higher-order contribution. ${ }^{7}$

In contrast to bosonic preheating, the appearance of classical turning points is unlikely. To make the classical scattering possible for the fermions, one has to put the four turning points together on the real axis of $t$, and such a scenario seems unreasonable.

In the above, we considered a scenario in which two states do not cross (or cross but the linear approximation is no longer valid), and found that the scenario leads to the scattering with higher potential. Alternatively, genuine higher dimensional interaction $\left(m_{D} \propto \phi^{n}, n \geq 2\right)$ can be used to introduce higher potential. The latter is what we have discussed previously for bosons.

The EWKB has been a powerful tool in understanding the physics behind the equations, especially when it is difficult to find the exact solution in terms of special functions.

\section{Conclusions and discussions}

In this paper, we examined cosmological particle production caused by a time-dependent background using the EWKB and the Stokes phenomena. Our focus was higher-dimensional

\footnotetext{
${ }^{7}$ We are not claiming that the contribution drastically changes the previous results $[27,28]$, since the situation considered in this paper is different from the previous calculation.
} 
interactions and the "classical" scattering process. The latter appears when the corresponding Schrödinger equation develops classical turning points on the real axis of time $(t)$. First, we examined a typical preheating scenario and found that amplification by the classical scattering is not possible. Then, using the EWKB, we developed simple calculational methods for preheating with the higher-dimensional interaction, and found that such (classical) amplification is indeed possible for the higher-dimensional interaction. Finally, we analyzed fermionic preheating mentioning the two distinctive sources of the higher-dimensional interaction and the possibility of the classical turning points.

In our future works, we are going to analyze in detail the conditions for asymmetric particle production [16, 29-33], using the ideas developed in this paper. Since baryogenesis requires B-violating interaction, and the interaction has to play a significant role in generating the asymmetry, the equations have to be multi-component (i.e, higher-order after decoupling). From the mathematical side, the Stokes phenomena of such higher-order equations have been analyzed for decades using the EWKB, in which virtual turning points are found to be crucial [24]. While on the cosmological side, at least when compared with the mathematical developments of the same period, the discussions of particle production and baryogenesis have been quite ad-hoc. In our future works, we are going to solve the Stokes phenomena of multi-component equations when interactions (i.e, off-diagonal elements) are time-dependent, to show explicitly how the asymmetry appears in cosmological particle creation with time-dependent backgrounds [16]. The EWKB considered in this paper is useful for calculating the connection formulae of complicated differential equations, which cannot be solved simply using special functions, and such equations may often appear in the cosmological calculation of the baryon asymmetry.

\section{Acknowledgments}

The authors would like to thank Nobuhiro Maekawa for collaboration on the early stages of this work. SE was supported by the Sun Yat-sen University Science Foundation.

\section{A Comparison with the steepest descent method}

In this section, we are going to review the calculation of the steepest descent method and compare it with the EWKB. The steepest descent method was applied to the cosmological particle production in refs. [11-13]. One can easily check that these two are consistent with each other.

Let us consider solving the following equation of motion:

$$
\ddot{\chi}_{k}+\left[k^{2}+m_{0}^{2}+\frac{g_{2 n}^{2}}{M_{*}^{2(n-1)}}(v t)^{2 n}\right] \chi_{k}=0 .
$$

This equation reproduces eq. (3.5);n=1 gives $g_{4}=0$ and $n=2$ gives $g_{2}=0$. If the adiabatic condition, which corresponds to

$$
\frac{\dot{\omega}_{k}}{\omega_{k}^{2}} \ll 1
$$


where

$$
\omega_{k} \equiv \sqrt{k^{2}+m_{0}^{2}+\frac{g_{2 n}^{2}}{M_{*}^{2(n-1)}} v^{2 n} t^{2 n}}
$$

is satisfied, eq. (A.1) has the (conventional) WKB solution given by

$$
\chi_{k}=\frac{\alpha_{k}}{\sqrt{2 \omega_{k}}} e^{-i \int_{-\infty}^{t} d t^{\prime} \omega_{k}\left(t^{\prime}\right)}+\frac{\beta_{k}}{\sqrt{2 \omega_{k}}} e^{+i \int_{-\infty}^{t} d t^{\prime} \omega_{k}\left(t^{\prime}\right)}
$$

where $\alpha_{k}, \beta_{k}$ are the complex constants that satisfies

$$
\left|\alpha_{k}\right|^{2}-\left|\beta_{k}\right|^{2}=1
$$

The WKB solution may have time-dependent coefficients such as

$$
\alpha_{k} \rightarrow \alpha_{k}(t), \quad \beta_{k} \rightarrow \beta_{k}(t)
$$

where the additional constraint

$$
0=\frac{\dot{\alpha}_{k}}{\sqrt{2 \omega_{k}}} e^{-i \int_{-\infty}^{t} d t^{\prime} \omega_{k}\left(t^{\prime}\right)}+\frac{\dot{\beta}_{k}}{\sqrt{2 \omega_{k}}} e^{+i \int_{-\infty}^{t} d t^{\prime} \omega_{k}\left(t^{\prime}\right)}
$$

has to be satisfied. Substituting the representation (A.4) into (A.1) with the above constraint, one can obtain

$$
\begin{aligned}
& \dot{\alpha}_{k}=\beta_{k} \frac{\dot{\omega}_{k}}{2 \omega_{k}} e^{+2 i \int_{-\infty}^{t} d t^{\prime} \omega_{k}}, \\
& \dot{\beta}_{k}=\alpha_{k} \frac{\dot{\omega}_{k}}{2 \omega_{k}} e^{-2 i \int_{-\infty}^{t} d t^{\prime} \omega_{k}} .
\end{aligned}
$$

Since we are considering a zero-particle state as the initial state, the initial conditions are given by

$$
\alpha_{k}(-\infty)=1, \quad \beta_{k}(-\infty)=0 .
$$

At $t=+\infty, \beta_{k}$ may be non-zero if particle production happens around $t=0$. Assuming that $\left|\beta_{k}(+\infty)\right|^{2} \ll 1$, one can approximate $\alpha_{k} \sim 1$ during the whole process. Then, $\beta$ at the final state can be estimated from (A.9) as

$$
\beta_{k}(+\infty)=\int_{-\infty}^{+\infty} d t \frac{\dot{\omega}_{k}(t)}{2 \omega_{k}(t)} \exp \left[-2 i \int_{-\infty}^{t} d t^{\prime} \omega_{k}\left(t^{\prime}\right)\right] .
$$

Now, one can evaluate the integral of eq. (A.11) using the steepest descent method. To understand the integral, we focus on

$$
\frac{\dot{\omega}_{k}}{2 \omega_{k}}=\frac{n}{2} \frac{t^{2 n-1}}{t_{k}^{2 n}+t^{2 n}}
$$

where

$$
t_{k} \equiv\left(\frac{k^{2}+m_{0}^{2}}{g_{2 n}^{2} M_{*}^{2}}\right)^{1 / 2 n} \frac{M_{*}}{v} .
$$




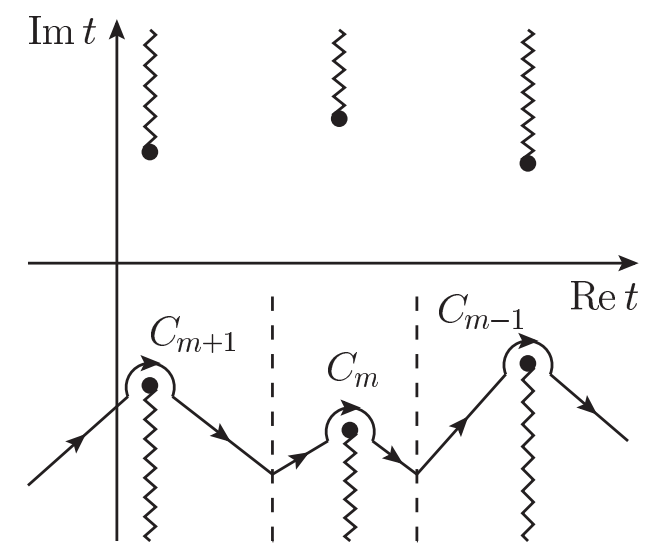

Figure 14. The path of integration on the $t$ complex plane. (This figure is the same as the one used in ref. [12].) The dots show the poles which are also saddle points, and the wavy lines show the branch cuts.

After analytic continuation, (A.12) has $2 n$ poles at

$$
t=t_{k} e^{i \pi(m+1 / 2) / n} \equiv t_{m}
$$

for $m=0,1, \cdots, 2 n-1$. At the neighborhood of $t \sim t_{m}$, one can obtain

$$
\begin{aligned}
\frac{\dot{\omega}_{k}}{2 \omega_{k}} & =\frac{1}{4} \frac{1}{t-t_{m}}\left(1+\mathcal{O}\left(t-t_{m}\right)\right) \\
\int_{-\infty}^{t} d t^{\prime} \omega_{k}\left(t^{\prime}\right) & =\int_{-\infty}^{t_{m}} d t^{\prime} \omega_{k}\left(t^{\prime}\right)+\frac{2 \sqrt{2 n} g_{2 n} v^{n} t_{m}^{n-1 / 2}}{3 M_{*}^{n-1}}\left(t-t_{m}\right)^{3 / 2}+\mathcal{O}\left(\left(t-t_{m}\right)^{5 / 2}\right) .
\end{aligned}
$$

Choosing the integral path in the lower half, which corresponds to choosing $m=n, n+$ $1, \cdots, 2 n-1$, we find that eq. (A.11) can be rewritten as

$$
\beta_{k}(+\infty) \sim \sum_{m=n}^{2 n-1} U_{m} \exp \left[-2 i \int_{-\infty}^{t_{m}} d t^{\prime} \omega_{k}\left(t^{\prime}\right)\right],
$$

where

$$
U_{m} \equiv \frac{1}{4} \int_{C_{m}} \frac{d t}{t-t_{m}} \exp \left[-i \frac{4 \sqrt{2 n} g_{2 n} v^{n} t_{m}^{n-1 / 2}}{3 M_{*}^{n-1}}\left(t-t_{m}\right)^{3 / 2}\right] .
$$

$C_{m}$ denotes the path approaching to and going around the pole at $t=t_{m}$ on the steepest descent path (and leaves the pole). See figure 14. If we take the angle going around the pole to be $4 \pi / 3$, the outward-going path becomes the steepest descent path. Then, we can obtain

$$
U_{m} \sim \frac{i \pi}{3}
$$

Using the above results, the distribution can be calculated as

$$
\left|\beta_{k}\right|^{2} \sim \frac{\pi^{2}}{9} \sum_{m, m^{\prime}=n}^{2 n-1} \exp \left[-2 i\left(\Omega_{m}-\Omega_{m^{\prime}}^{*}\right)\right]
$$


where

$$
\begin{aligned}
\Omega_{m} & \equiv \int_{0}^{t_{m}} d t^{\prime} \omega_{k}\left(t^{\prime}\right) \\
& =\sqrt{k^{2}+m_{0}^{2}} t_{k} e^{i \pi(m+1 / 2) / n} \times \frac{\Gamma\left(\frac{3}{2}\right) \Gamma\left(1+\frac{1}{2 n}\right)}{\Gamma\left(\frac{3}{2}+\frac{1}{2 n}\right)} .
\end{aligned}
$$

Especially, in the case of $n=1$, we can obtain

$$
\begin{aligned}
\left|\beta_{k}(+\infty)\right|^{2} & \sim \frac{\pi^{2}}{9} e^{-2 i\left(\Omega_{1}-\Omega_{1}^{*}\right)}=\frac{\pi^{2}}{9} \exp \left[-\pi \frac{k^{2}+m_{0}^{2}}{g_{2} v}\right] \\
& =\frac{\pi^{2}}{9} e^{-2 K_{u d}}
\end{aligned}
$$

where $K_{u d}$ is defined in (2.26). Hence, this result reproduces the results shown in 2.2 except for the prefactor $\pi^{2} / 9 \sim 1.097$. This extra factor seems to be due to the approximation by the steepest descent method.

For the case of $n=2$, we can obtain

$$
\begin{aligned}
\left|\beta_{k}(+\infty)\right|^{2} & \sim \frac{\pi^{2}}{9}\left(e^{-2 i\left(\Omega_{2}-\Omega_{2}^{*}\right)}+e^{-2 i\left(\Omega_{3}-\Omega_{3}^{*}\right)}+e^{-2 i\left(\Omega_{2}-\Omega_{3}^{*}\right)}+e^{-2 i\left(\Omega_{3}-\Omega_{2}^{*}\right)}\right) \\
& =\frac{\pi^{2}}{9} \cdot 2 e^{-2.47 \kappa_{4}^{3 / 2}}\left(1+\cos \left(2.47 \kappa_{4}^{3 / 2}\right)\right) \\
& =\frac{\pi^{2}}{9} \cdot 2 e^{-2 K_{A B}}\left(1+\cos \left(2 K_{A B}\right)\right)
\end{aligned}
$$

where $\kappa_{4}$ and $K_{A B}$ are defined in (3.11) and (3.15), respectively. This result also reproduces the analytic result by the EWKB method except for the prefactor.

Open Access. This article is distributed under the terms of the Creative Commons Attribution License (CC-BY 4.0), which permits any use, distribution and reproduction in any medium, provided the original author(s) and source are credited.

\section{References}

[1] J.H. Traschen and R.H. Brandenberger, Particle production during out-of-equilibrium phase transitions, Phys. Rev. D 42 (1990) 2491.

[2] L. Kofman, A.D. Linde and A.A. Starobinsky, Towards the theory of reheating after inflation, Phys. Rev. D 56 (1997) 3258 [hep-ph/9704452] [inSPIRE].

[3] L. Kofman, A.D. Linde, X. Liu, A. Maloney, L. McAllister and E. Silverstein, Beauty is attractive: moduli trapping at enhanced symmetry points, JHEP 05 (2004) 030 [hep-th/0403001] [INSPIRE].

[4] G.N. Felder, J. García-Bellido, P.B. Greene, L. Kofman, A.D. Linde and I. Tkachev, Dynamics of symmetry breaking and tachyonic preheating, Phys. Rev. Lett. 87 (2001) 011601 [hep-ph/0012142] [INSPIRE].

[5] M.V. Berry and K.E. Mount, Semiclassical approximations in wave mechanics, Rept. Prog. Phys. 35 (1972) 315. 
[6] V.L. Pokrovskii and I.M. Khalatnikov, On the Problem of Above-Barrier Reflection of High-Energy Particles, Zh. Eksp. Teor. Fiz. 40 (1961) 1713 [Sov. Phys. JETP 13 (1961) 1207].

[7] E. Brezin and C. Itzykson, Pair production in vacuum by an alternating field, Phys. Rev. D 2 (1970) 1191.

[8] J. Schwinger, On gauge invariance and vacuum polarization, Phys. Rev. 82 (1951) 664.

[9] S. Shakeri, M.A. Gorji and H. Firouzjahi, Schwinger Mechanism During Inflation, Phys. Rev. D 99 (2019) 103525 [arXiv: 1903.05310] [INSPIRE].

[10] H. Kitamoto, No-go theorem of anisotropic inflation via Schwinger mechanism, arXiv:2010.10388 [INSPIRE].

[11] D.J.H. Chung, Classical Inflation Field Induced Creation of Superheavy Dark Matter, Phys. Rev. D 67 (2003) 083514 [hep-ph/9809489] [INSPIRE].

[12] S. Enomoto, S. Iida, N. Maekawa and T. Matsuda, Beauty is more attractive: particle production and moduli trapping with higher dimensional interaction, JHEP 01 (2014) 141 [arXiv: 1310.4751] [INSPIRE].

[13] S. Enomoto, N. Maekawa and T. Matsuda, Preheating with higher dimensional interaction, Phys. Rev. D 91 (2015) 103504 [arXiv:1405.3012] [INSPIRE].

[14] A. Voros, The return of the quartic oscillator. The complex WKB method, Ann. Inst. Henri Poincaré 39 (1983) 211.

[15] Résurgence de voros et périodes des courbes hyperelliptiques, Annales Inst. Fourier 43 (1993) 163.

[16] S. Enomoto and T. Matsuda, Kinematic Baryogenesis, to appear.

[17] G. Lazarides and C. Panagiotakopoulos, Smooth hybrid inflation, Phys. Rev. D 52 (1995) 559 [hep-ph/9506325] [inSPIRE].

[18] T. Matsuda, Remote Inflation: Hybrid-like inflation without hybrid-type potential, JCAP 07 (2009) 003 [arXiv:0904.2821] [INSPIRE].

[19] T. Matsuda, Thermal hybrid inflation in brane world, Phys. Rev. D 68 (2003) 047702 [hep-ph/0302253] [INSPIRE].

[20] T. Matsuda, Cosmological perturbations from inhomogeneous preheating and multi-field trapping, JHEP 07 (2007) 035 [arXiv:0707.0543] [INSPIRE].

[21] T. Matsuda, On the cosmological domain wall problem in supersymmetric models, Phys. Lett. B 436 (1998) 264 [hep-ph/9804409] [INSPIRE].

[22] H. Shen and H.J. Silverstone, Observations on the JWKB treatment of the quadratic barrier, Algebraic Analysis of Differential Equations (2008) 237.

[23] T. Aoki, T. Kawai, and T. Takei, The Bender-Wu analysis and the Voros theory. II, Adv. Stud. Pure Math. 54 (2009).

[24] N. Honda, T. Kawai and Y. Takei, Virtual Turning Points, Springer Japan (2015), DOI.

[25] T. Aoki and J. ichi Yoshida, Microlocal reduction of ordinary differential operators with a large parameter, Publ. Res. Inst. Math. Sci. 29 (1993) 959.

[26] M. Peloso and L. Sorbo, Preheating of massive fermions after inflation: Analytical results, JHEP 05 (2000) 016 [hep-ph/0003045] [INSPIRE]. 
[27] P.B. Greene and L. Kofman, Preheating of fermions, Phys. Lett. B 448 (1999) 6 [hep-ph/9807339] [INSPIRE].

[28] G.F. Giudice, M. Peloso, A. Riotto and I. Tkachev, Production of massive fermions at preheating and leptogenesis, JHEP 08 (1999) 014 [hep-ph/9905242] [INSPIRE].

[29] A. Dolgov, K. Freese, R. Rangarajan and M. Srednicki, Baryogenesis during reheating in natural inflation and comments on spontaneous baryogenesis, Phys. Rev. D 56 (1997) 6155 [hep-ph/9610405] [INSPIRE].

[30] K. Funakubo, A. Kakuto, S. Otsuki and F. Toyoda, Charge generation in the oscillating background, Prog. Theor. Phys. 105 (2001) 773 [hep-ph/0010266] [INSPIRE].

[31] R. Rangarajan and D.V. Nanopoulos, Inflationary baryogenesis, Phys. Rev. D 64 (2001) 063511 [hep-ph/0103348] [INSPIRE].

[32] S. Enomoto and T. Matsuda, Asymmetric preheating, Int. J. Mod. Phys. A 33 (2018) 1850146 [arXiv: 1707.05310 ] [INSPIRE].

[33] S. Enomoto and T. Matsuda, Baryogenesis from the Berry phase, Phys. Rev. D 99 (2019) 036005 [arXiv: 1811.06197] [INSPIRE]. 